\title{
Breast cancer stem-like cells are sensitized to tamoxifen induction of self-renewal inhibition with enforced Let-7c dependent on Wnt blocking
}

\author{
XIN SUN ${ }^{1 *}$, CHONGWEN XU $^{2 *}$, GUODONG XIAO ${ }^{1 *}$, JINYING MENG $^{3}$, JICHANG WANG $^{4}$,

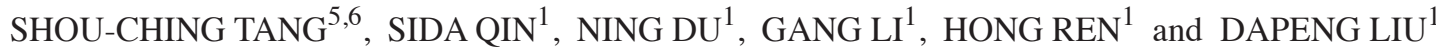 \\ ${ }^{1}$ Department of Thoracic Surgery and Oncology, The Second Department of Thoracic Surgery, Cancer Center; \\ ${ }^{2}$ Department of Otorhinolaryngology, The First Affiliated Hospital of Xi'an Jiaotong University, Xi'an, Shaanxi 710061; \\ ${ }^{3}$ Department of Surgery Oncology, The First People's Hospital of Xianyang City, Xianyang, Shaanxi 712000; \\ ${ }^{4}$ Department of Vascular and Endovascular Surgery, The First Affiliated Hospital of Xi'an Jiaotong University, Xi'an, \\ Shaanxi 710061; ${ }^{5}$ Solid Tumor Clinical Trials, Georgia Cancer Center, Medical College of Georgia, Augusta University, \\ Augusta, GA 30912, USA; ${ }^{6}$ Tianjin Medical University Cancer Institute and Hospital, Tianjin 300060, P.R. China
}

Received March 2, 2017; Accepted December 4, 2017

DOI: $10.3892 / \mathrm{ijmm} .2018 .3388$

\begin{abstract}
Let-7 microRNAs have been reported to have tumor suppressive functions; however, the effect of Let-7 when used in combination with chemotherapies is uncertain, but may have potential for use in clinical practice. In this study, we used RT-qPCR, western blot analysis, cell proliferation assay, flow cytometry analysis, immunohistochemistry (IHC) staining, luciferase assays, cell sorting analysis and xenografted tumor model to explore the role of Let-7 in the chemotherapy sensitivity of breast cancer stem cells. The findings of the current study indicated that Let-7 enhances the effects of endocrine therapy potentially by regulating the self-renewal of cancer stem cells. Let-7c increased the anticancer functions of tamoxifen and reduced the ratio of cancer stem-like cells (CSCs), sensitizing cells to therapy-induced repression in an estrogen receptor (ER)-dependent manner. Notably, Let-7 decreased the tumor formation ability of estrogen-treated breast CSCs in vivo and suppressed Wnt signaling, which further consolidated the previously hypothesis that Let-7 decreases the self-renewal ability, contributing to reduced tumor formation ability of stem cells. The suppressive effects exerted by Let-7 on stem-like cells involved Let-7c/ER/Wnt signaling, and the
\end{abstract}

Correspondence to: Professor Dapeng Liu or Professor Hong Ren, Department of Thoracic Surgery and Oncology, The Second Department of Thoracic Surgery, Cancer Center, The First Affiliated Hospital of Xi'an Jiaotong University, 277 West Yanta Road, Xi'an, Shaanxi 710061, P.R. China

E-mail: prof_ldp_oncology@126.com

E-mail: medicinedr.ren@gmail.com

*Contributed equally

Key words: Let-7 microRNAs, breast cancer, cancer stem-like cells, endocrine therapy, estrogen receptor, tamoxifen functions of Let-7c exerted with tamoxifen were dependent on ER. Taken together, the findings identified a biochemical and functional link between Let-7 and endocrine therapy in breast CSCs, which may facilitate clinical treatment in the future using delivery of suppressive Let-7.

\section{Introduction}

Breast cancer has the highest rate of cancer-associated mortality in women worldwide (1). Typically, patients with breast cancer are treated with chemotherapy, radiotherapy, surgeries, targeted therapy, and most importantly, the endocrine therapy, which may improve their prognosis. The long-term survival is often hindered by tumor recurrence, which is resistant to original therapy and causes the tumor treatment strategy to be changed. Anti-estrogen strategy has been widely used in clinical treatments, with anti-estrogen drugs or estrogen receptor (ER) antagonists, including tamoxifen, letrozole and anastrozole, as important breast cancer treatments $(2,3)$. Unfortunately, many patients are still either resistant to these drugs, or face serious side effects (4-6). Reducing drug-resistance and suppressing cancer malignancy is essential, and effective strategies to eradicate cancer as required (7).

MicroRNA (miRNA) Let-7a was previously identified to target stem-like cells in cancer via inhibition of ER $\alpha$ (8), and Let-7b acted as the indicator of therapy response. Notably, Let-7a may function as the key downstream activator in the process of upstream anti-oncogenes induced tumor inhibition (9-12). The role of Let-7c in cancer stem cells was previously explored, and the miRNA was demonstrated to reduce the tumor formation ability of cancer-stem like cells via negative regulation of estrogen receptor 1 (ESR1)/Wnt1 signaling pathway (13). However, its roles in regulation of endocrine therapy response have rarely been studied. The current study aimed to elucidate the potential functions of Let-7 in mediating the response of breast cancer stem-like cells (CSCs) to anti-endocrine agents, which may identify 
strategies to reduce therapy resistance of cancer cells, and avoid the long-term usage of endocrine drugs.

\section{Materials and methods}

Tissue samples. Sixty NSCLC samples and paired normal tumor-adjacent samples were obtained from the First Affiliated Hospital of Xi'an Jiaotong University, Xi'an, China. All samples without chemotherapy, immunotherapy or radiotherapy were collected between June 2008 and October 2014. This study was approved by Xi'an Jiaotong University Ethics Committee and written informed consent was obtained from all patients. They consisted of 43 males and 17 females with median age of 60 years (age range, 55-81 years). Tumor tissue and matched normal tumor-adjacent tissue specimens were obtained at surgery and immediately stored in formalin solution to make paraffin-embedded specimens for immunohistochemistry.

Cell culture and lentiviral infection. ER-positive breast cancer cell lines (MCF-7 and T47-D) and 293T cells were purchased from American Type Culture Collection (Manassas, VA, USA) and maintained at Center for Translational Medicine, First Affiliated Hospital of Xi'an Jiaotong University (Xi'an, China). Cells culturing and spheres non-adjacent seeding were performed as previously did $(10,13)$. In brief, cells were cultured in high glucose Dulbecco's Modified Eagle's Medium (DMEM; HyClone, Logan, UT, USA) supplemented with $10 \%$ fetal bovine serum (FBS; Gibco; Thermo Fisher Scientific, Inc., Waltham, MA, USA). For tumor sphere culture, dissected primary breast tumor cells were plated in ultra-low attachment dishes (Corning Incorporated, Corning, NY, USA) without fetal bovine serum to determine their ability to form primary mammospheres, and the obtained mammospheres were disaggregated into single cells using Accutase (StemPro; Gibco; Thermo Fisher Scientific, Inc.) and trypsin-EDTA (0.05\%; Gibco; Thermo Fisher Scientific, Inc.), and then they were re-suspended to acquire the next generation of spheres. Lentivirus-mediated Let-7c overexpression or ESR1 short hairpin RNA (shRNA target sequence, 5'-AGGGAGAATGTTGAAGCACAA-3') mediated knockdown in MCF-7 and T47-D cells was performed as described and validated in a previous study (13).

Reverse transcription-quantitative polymerase chain reaction $(R T-q P C R)$. Total RNA was extracted using TRIzol reagent (Life Technologies; Thermo Fisher Scientific, Inc.) from clinical tissues and cultured cells, as previously described $(8,13,14)$. The first strand cDNA was synthesised using SuperScript III First-strand synthesis SuperMix (Invitrogen; Thermo Fisher Scientific, Inc.). RT-qPCR was performed using Powerup SYBR-Green Master Mix (Applied Biosystems; Thermo Fisher Scientific, Inc.) to detect Let-7c. The small nuclear RNA U6 was used as endogenous control. The primer sequences were as follows: Let-7c forward,5'-GCCGCTGAGGTAGTAGG TTGTAT-3' and reverse, 5'-GTGCAGGGTCCGAGGT-3'; U6 forward, 5'-CTCGCTTCGGCAGCACA-3' and reverse, 5'-AACGCTTCACGAATTTGCGT-3'. Expression levels were calculated and presented following the $2^{-\Delta \Delta \mathrm{Ct}}$ method $[\Delta \Delta \mathrm{Ct}=\Delta \mathrm{Ct}$ (positive) $-\Delta \mathrm{Ct}($ control) $](15-17)$.
Table I. Statistical analysis of tumor growth of stem cells from different groups in immune compromised mice ( $\mathrm{mm})$.

\begin{tabular}{|c|c|c|c|c|}
\hline \multirow[b]{2}{*}{ Day } & \multicolumn{2}{|c|}{ Mean $\pm \mathrm{SE}(\mathrm{mm})$} & \multirow[b]{2}{*}{ F-value } & \multirow[b]{2}{*}{ P-value } \\
\hline & Scramble & Let-7c & & \\
\hline 10 & $27.08 \pm 4.72$ & $16.28 \pm 1.27$ & 0.0263 & 0.1374 \\
\hline 14 & $62.66 \pm 16.89$ & $32.87 \pm 2.10$ & 0.001 & 0.1705 \\
\hline 18 & $121.08 \pm 26.62$ & $67.96 \pm 5.55$ & 0.010 & 0.1049 \\
\hline 22 & $173.93 \pm 30.24$ & $114.12 \pm 6.02$ & 0.009 & 0.0996 \\
\hline 26 & $261.53 \pm 37.04$ & $160.19 \pm 8.93$ & 0.017 & 0.0477 \\
\hline 30 & $398.34 \pm 38.88$ & $228.56 \pm 11.24$ & 0.034 & 0.0110 \\
\hline
\end{tabular}

SE, standard error.

Immunohistochemistry (IHC). Formalin-fixed and paraffinembedded sections (5- $\mu$ m-thick) from mice tumor tissues were deparaffinized in xylene and rehydrated with descending concentrations of ethanol (100, 95, 80 and 70\%). Antigen retrieval was performed by heating the sections in $0.01 \mathrm{M}$ citrate buffer at $95^{\circ} \mathrm{C}$ for $10 \mathrm{~min}$. Endogenous peroxidase was quenched by treatment with $0.3-3 \% \mathrm{H}_{2} \mathrm{O}_{2}$ and $100 \%$ methanol for $10 \mathrm{~min}$ at room temperature (RT). The sections were then blocked by applying 1-2 drops of $10 \%$ goat serum (ZSGB-BIO, Beijing, China) for $30 \mathrm{~min}$. After incubation with diluted primary antibody to ESR1 (1:100; sc-7207) and Wnt1 (1:200; sc-6280; both from Santa Cruz Biotechnology, Inc., Dallas, TX, USA), and $\beta$-catenin (1:200; cat. no. 9562; Cell Signaling Technology, Inc., Danvers, MA, USA) at $4^{\circ} \mathrm{C}$ overnight in a closed incubation chamber, the sections were then washed in PBS and incubated with orseradish peroxidase (HRP)-conjugated secondary antibody at RT for $1 \mathrm{~h}$.

Western blot analysis. Total protein were extracted and transferred as previously described $(14,18)$. In brief, total protein was extracted using radioimmunoprecipitation lysis buffer (Bioss, Co., Beijing, China), with protease and phosphatase inhibitor (x100; cat. no. 5872; Cell Signaling Technology, Inc.). The concentration of total proteins was quantified using Protein Quantification kit (BCA; ab102536; Abcam, Cambridge, UK). The protein lysates were electrophoreted and resolved in $10 \%$ SDS-PAGE, then transferred to nitrocellulose membranes. After blocking with $5 \%$ fat-free milk, the membranes were incubated at $4^{\circ} \mathrm{C}$ overnight with specific primary antibodies to ESR1 (1:2,000, sc-87; Santa Cruz Biotechnology, Inc.), t-cell factor 4 (tcf-4; 1:2,000; cat. no. 05-511; EMD Millipore, Billerica, MA, USA), oct-4 (1:1,000; sc-5279; Santa Cruz Biotechnology, Inc.), Sox-2 (1:3,000; cat. no. 2748) and vinculin $(1: 2,000$; cat. no. 4650 ; both from Cell Signaling Technology, Inc.); the secondary antibodies conjugated with horseradish peroxidase (1:3,000-1:5,000; cat. nos. 7071 and 7072; Santa Cruz Biotechnology, Inc.) were incubated for $1 \mathrm{~h}$ at room temperature.

Proliferation detection. The detection of cell proliferation was performed as previously reported $(8,10)$. Briefly, cells of each group were adjusted to $5 \times 10^{3}$ cells/well in a volume 
of $100 \mu 1$ medium in 96-well plates, and then cultured with $20 \mu \mathrm{l} \mathrm{MTT}(5 \mathrm{mg} / \mathrm{ml})$ at $37^{\circ} \mathrm{C}$ for $4 \mathrm{~h}$, the crystals of formazan was dissolved in dimethyl sulfoxide. The optical density was detected by spectrophotometer at $492 \mathrm{~nm}$.

Flow cytometry analysis of cycle apoptosis and cell cycle. Flow cytometry analysis for apoptosis and cycles was performed as described previously $(8,19,20)$. For apoptosis ratio analysis, cells $\left(5 \times 10^{4} / \mathrm{ml}\right)$ in the logarithmic phase were collected and cultured in 6-well dishes at $1 \times 10^{5}$ cells/well. The cells were suspended in $500 \mu \mathrm{l} 1 \mathrm{X}$ binding buffer, and then $5 \mu \mathrm{l}$ Annexin V-fluorescein isothiocyanate and $5 \mu \mathrm{l}$ propidium iodide (PI; $20 \mu \mathrm{g} / \mathrm{ml}$; both from BD Biosciences, Franklin Lakes, NJ, USA) were added. For cell cycle analysis, cancer cells were cultured and adjusted to $5 \times 10^{4} / \mathrm{ml}$ in 6 -well dish, and then fixed with ice-cold $1 \mathrm{ml}$ of $75 \%$ ethanol for $24 \mathrm{~h}$ at $4^{\circ} \mathrm{C}$. Fixed cells were stained with $150 \mu \mathrm{l} \mathrm{PI}$ and $150 \mu \mathrm{l}$ RNA enzyme inhibitor for $30 \mathrm{~min}$ at $37^{\circ} \mathrm{C}$. The cells were analyzed using a flow cytometer within $1 \mathrm{~h}$ of incubation and all analyses were performed in triplicate.

Fluorescence-activated cell sorting analysis and sphere forming assay. The LSR II flow cytometer (BD Pharmingen, San Diego, CA, USA) was used to analyze and separate CSCs based on cell labeling and fluorescence-activated sorting. The activated ALDEFLUOR $^{\mathrm{TM}}$ reagent and diethylaminobenzaldehyde purchased from Stemcell Technologies, Inc. (Vancouver, BC, Canada) were used to isolate aldehyde dehydrogenase (ALDH) ${ }^{+}$ cells (21-23). The ratios of ALDH1 ${ }^{+}$stem cells in different groups were analyzed. The ratios of aldH $1+$ stem cells in different groups were analyzed. For sphere forming assay, CSCs (1000cells per/ well) were seeded in 6-well ultra-low cluster plates (Corning Inc., Corning, NY) for one week to obtain first generation after being given different treatment. Spheres were cultured in DMEM/F12 serum-free medium (Invitrogen) supplemented with 2\% B27 (BD Biosciences, CA), 20ng/ml epidermal growth factor (EGF, Sigma), 20ng/ml basic fibroblast growth factor (bFGF, Sigma), $0.4 \%$ BSA and $4 \mu \mathrm{g} / \mathrm{ml}$ insulin (Sigma). The number of spheres was counted under a microscope.

Luciferase assays and transient transfection. MCF-7 and T47-D cells were seeded in 24-well plates with $1 \times 10^{5}$ cells/well, and only cells growing in the log phase at a passage number $\leq 15$ were used. Cells were transfected with Let-7c mimic and Renilla luciferase plasmid containing a TCF-4-responsive promoter when at 50-70\% confluence ratio using Lipofectamine ${ }^{\circledR} 2000$ (Invitrogen; Thermo Fisher Scientific, Inc.), non-transfected and non-treated cells were set as control, as described previously $(9,11)$. Each group included three replicates, and independent triplicate experiments were performed. Specifically, the cells were collected with cell lysis buffer after transfection for $24 \mathrm{~h}$, and Renilla luciferase activity was detected using the Pierce ${ }^{\circledR}$ Renilla Luciferase Flash Assay kit (no. 16164; Thermo Fisher Scientific, Inc.), following the manufacturer's instructions. Cell lysate $(20 \mu \mathrm{l})$ and $50 \mu \mathrm{l}$ working solution were added to one column, and a Turner Biosystems TD-20/20 luminometer (Turner Biosystems; Thermo Fisher Scientific, Inc.) was used to detect the light output (prime the pump prior to injections with working solution). Firefly luciferase activity was normalized to Renilla luciferase activity.
Nude mice study. MCF-7 stem cells transfected with Let-7c mimic and control were pretreated with $10 \mathrm{nM}$ estrogen for $24 \mathrm{~h}$ and implanted subcutaneously into BALB/c mice $(\mathrm{n}=5$; age, 5 weeks) supplied by the Experimental Animal Center, College of Medicine, Xi'an Jiaotong University [license no. SCXK (shan) 2007-001] (10,18,24,25). Long-release estradiol pellets (Innovative Research of America, Sarasota, FL, USA) were implanted the day before inoculation. Tumor growth was measured by a digital caliper every 5 days for 5 weeks. Tumor weight was measured after cell implantation. Animal experimental researches followed the internationally recognized guidelines. Animal studies were approved by the Medical College of Xi'an Jiaotong University Animal Ethics Board. Protocols were conducted in accordance with the guidance for the Care and Use of Laboratory Animals, established by the Ministry of Science and Technology of China (Beijing, China).

Statistical analysis. All statistical analyses were performed using SPSS version 16. All data are presented as mean \pm standard errorof the mean(SEM). Statisticalanalyses were conduced using Student's t-test and one-way ANOVA. Kruskal-Wallis test was used to analyze the intensity of ALDH, Wnt1 and TCF-4 in different phases of breast cancer tissues. $\mathrm{P}<0.05$ was considered to indicate a statistically significant difference.

\section{Results}

Stem-like cells and Wnt signaling activation are associated with advanced progression of patients with ER-positive breast cancer. Clinical data of tissues from patients of breast cancer of ER status were retrospectively analyzed, and ALDH1 was stained using IHC to determine the ratio of stem-like cells in cancer specimens. The ratio of $\mathrm{ALDH}^{+}$cells increased significantly with advanced cancer stages (Fig. 1A, left). Increased Wnt signaling activation, represented by staining for Wnt1 (Fig. 1A, middle panel) and TCF-4 (Fig. 1A, right panel), was associated with the later phases of breast cancer.

Estrogen induction of stimulation of self-renewal ability. To identify the oncogenic role of estrogen in regulations of isolated stem cells, intermittent addition of estrogen was used during the culture of non-adjuvant spheres. The intermittent treatment of estrogen in certain generations assured its effects on either sphere formation, or sphere cell proliferation. Estrogen $(10 \mathrm{nM})$ was added into the medium continuously in every generation of spheres culturing (group b), or was added on the 1st, 3rd and 5th generation (group a), as illustrated in Fig. 1B. Estrogen treatment at the 1st generation increased the ratio of stem cells, affecting the self-renewal, and no significant difference was detected between groups a and b. However, the difference was notified and identified in the 3rd and the 5th generation, which was resulted from different strategy of estrogen addition in the 2nd and 4th generation (Fig. 1C), demonstrating the effects of estrogen in controlling the self-renewal ability of stem-like cells.

Our previous study demonstrated that Let-7c inhibited the ratio of $\mathrm{ALDH}^{+}$cells in breast cancer cells via suppressing the function of estrogen induction of Wnt signaling activation (13). The results of the current study demonstrated that the inhibition of tamoxifen on self-renewal ability of CSCs became greater when the concentration increased (data not 
A
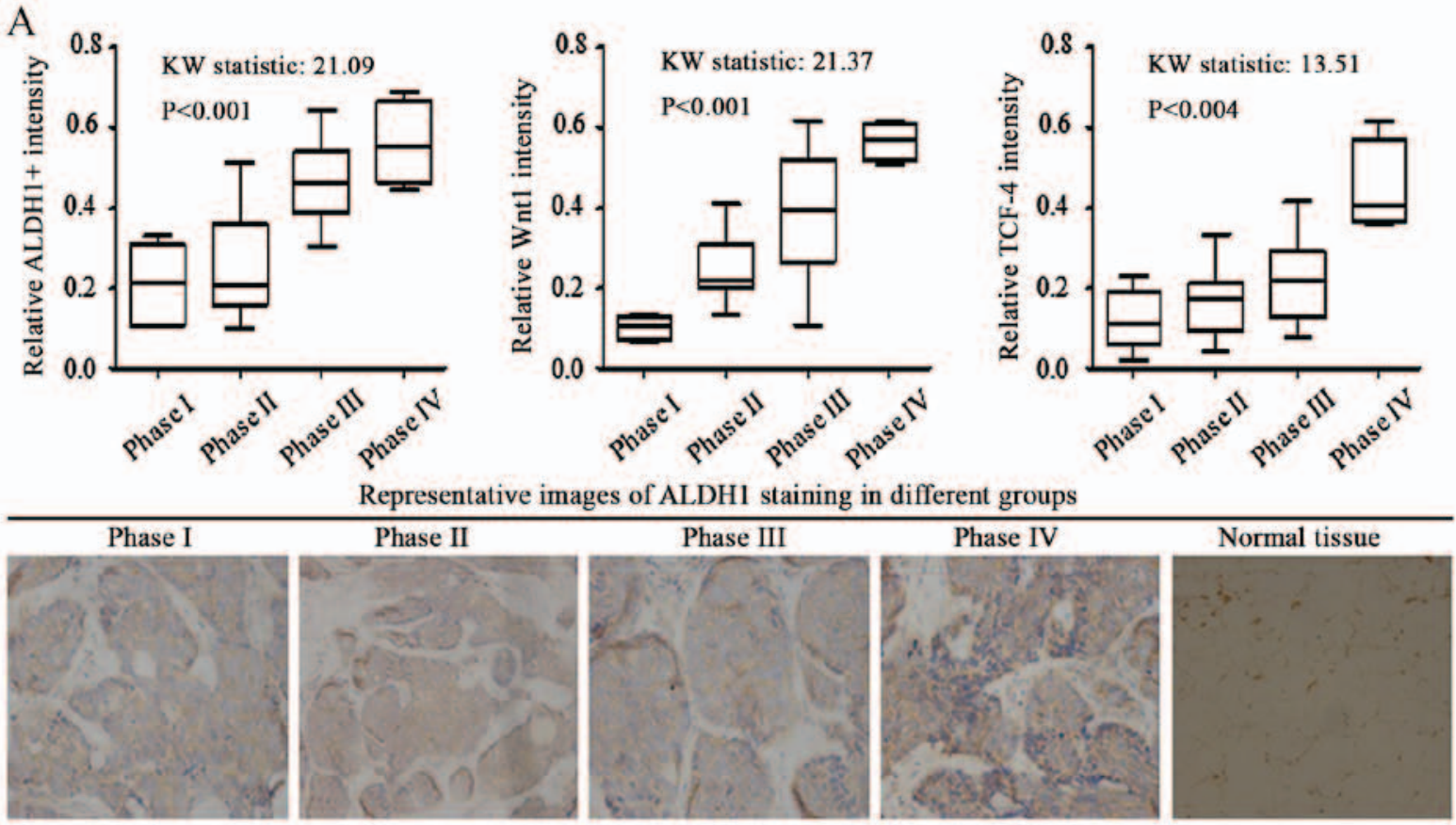

$\mathrm{B}$

B Estrogen

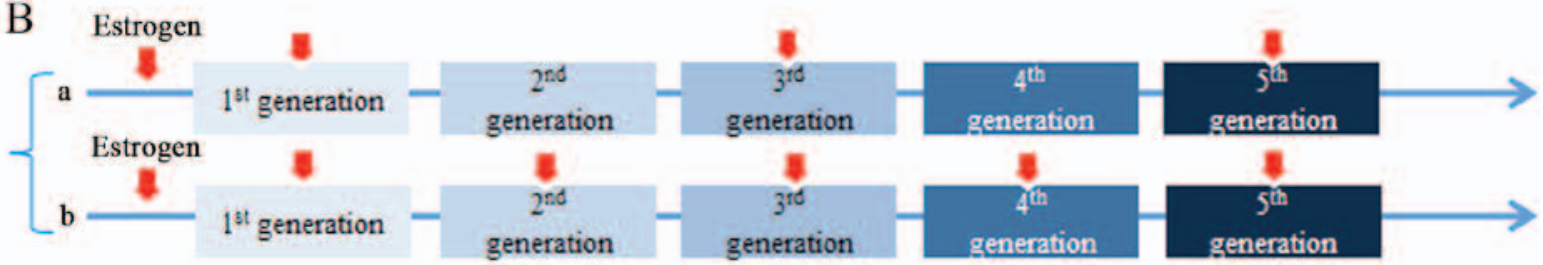

C
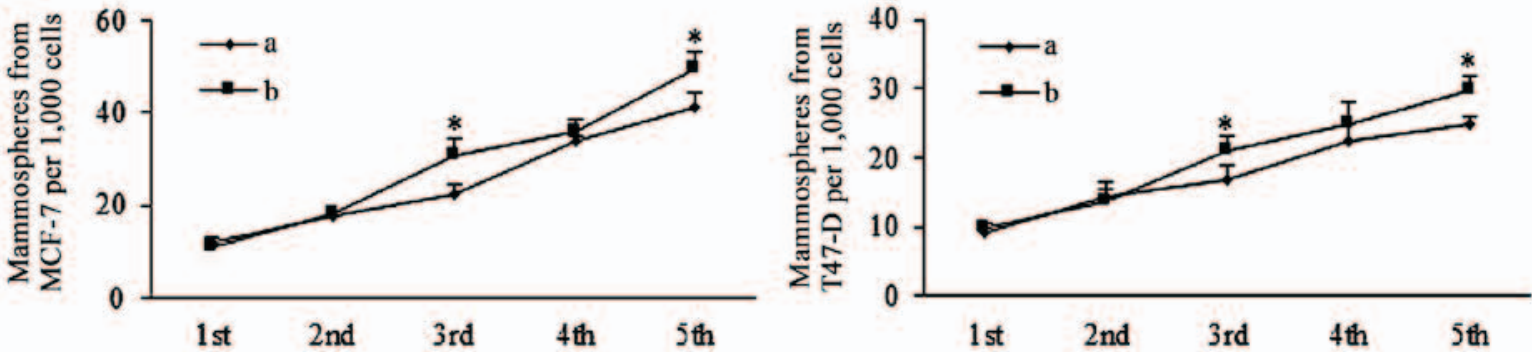

D
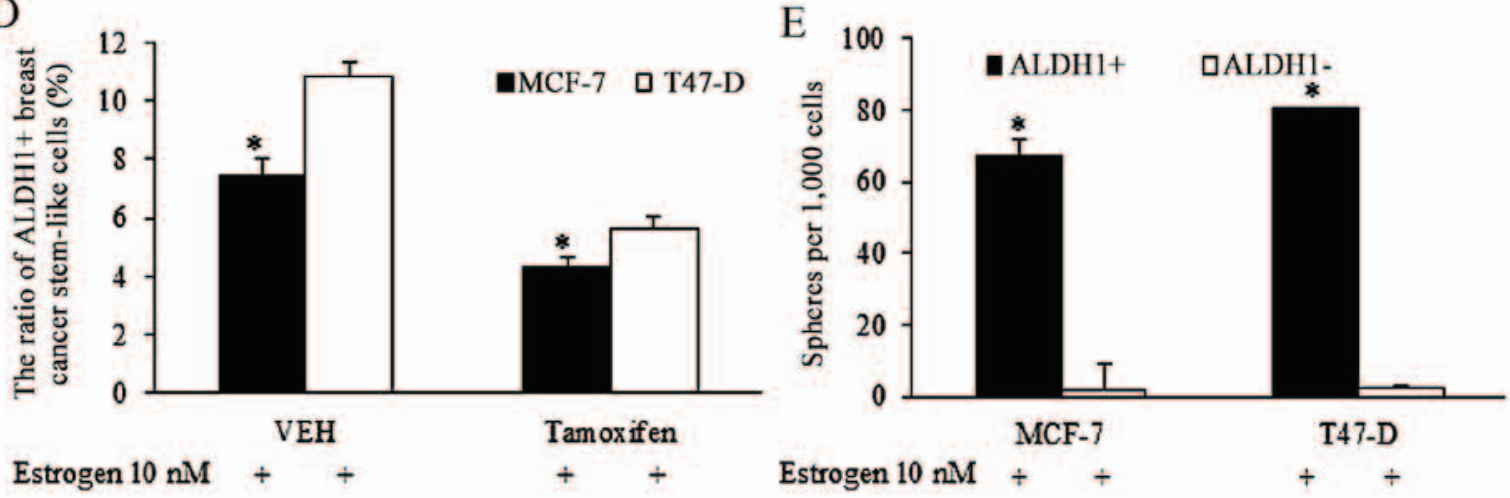

Figure 1. Stem-like cells are associated with progressed cancer stages and Wnt signaling activation in breast cancer of estrogen receptor (ER) status. (A) Clinical studies of tissues from patients of breast cancer of ER status, ALDH1 was stained for illustrating the ratio of stem-like cells in cancer specimens, and the ratio of ALDH1-positive cells increased with advanced cancer stages (left panel). The Wnt signaling activation was corresponding to the later phase of breast cancer, identified by Wnt1 (middle panel) and TCF-4 (right panel) immunohistochemical staining (original magnification, x400). (B) Description of estrogen addition strategy in sphere-formation assays, the $10 \mathrm{nM}$ estrogen was added into the medium continuously in every generation of spheres culture, or was added in pretreatment medium, and the 1st, 3rd and 5th generation. (C) Comparison of effect of different estrogen addition strategy on mammosphere formation ability of breast cancer stem cells, and the existence of estrogen stimulated the self-renewal ability of stem cells of breast cancer. ${ }^{*} \mathrm{P}<0.05$ vs. group a. (D) Anti-estrogenic tamoxifen decreased the ratio of ALDH1-positive cells in MCF-7 and T47-D cells. ${ }^{*} \mathrm{P}<0.05$. (E) ALDH1-positive cells harbored increases sphere formation ability, compared with ALDH1-negative cells. Statistical significance was evaluated by using Kruskal-Wallis test or analysis of variance. ${ }^{*} \mathrm{P}<0.05$ vs. ALDH ${ }^{*}$ ALDH, aldehyde dehydrogenase; TCF-4, T-cell factor $4 ; \mathrm{VEH}$, vehicle ( $\mathrm{n}=3$ repeats with similar results. Values are presented as the means \pm SEM) 
A

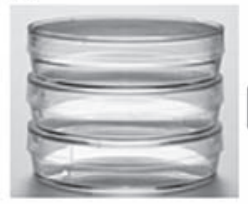

Adhering culture

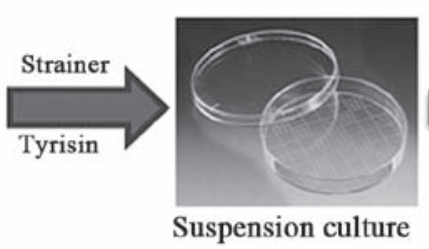

$\mathrm{C}$

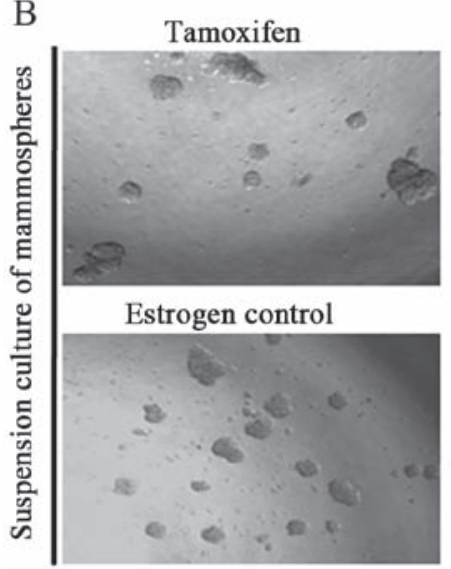

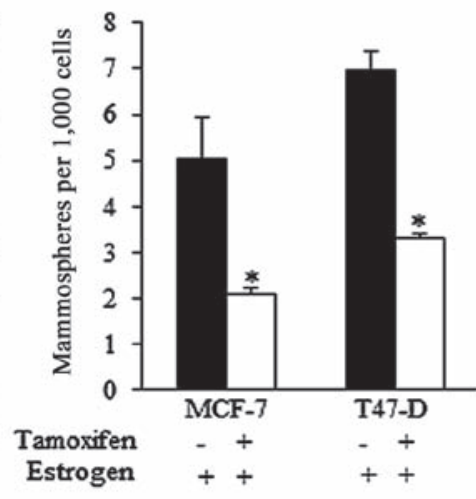
Estrogen

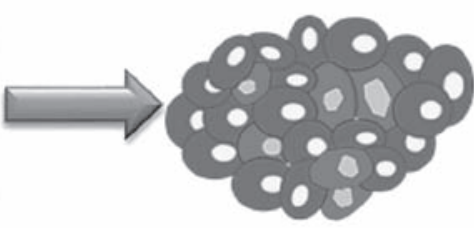

$\mathrm{D}$

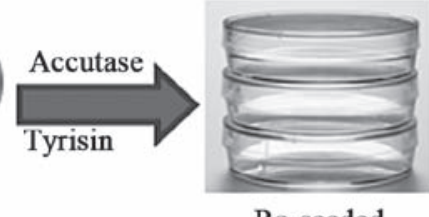

Re-seeded adhering culture

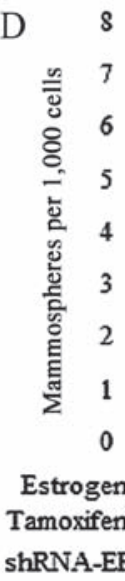

Let-7c

Figure 2. Breast cancer stem-like cells isolated from spheres were sensitized to tamoxifen-induced self-renewal inhibition. (A) Strategy of acquiring mammospheres and subsequent analysis of the apoptosis ratio of stem-like cells. The stem-like cells were digested by trypsin/accutase enzyme and then reseeded. (B) Representative images of spheres from different groups. (C) Tamoxifen decreased the ratio of mammospheres acquired from estrogen-treated breast cancer cells. (D) Let-7c mimic transfection enhanced the tamoxifen functions, helped to decrease the spheres number greatly, however. ER inhibition using shRNA did not exhibit improved anticancer effects on the group treated with combined tamoxifen and Let-7c, compared to the control group with normal ER status. shRNA, short hairpin RNA; ER, estrogen receptor ( $n=3$ repeats with similar results. Values are presented as the means \pm SEM).

shown), and $10^{-5} \mathrm{~mol} / \mathrm{l}$ tamoxifen reduced the ratio of $\mathrm{ALDH} 1^{+}$ cells (Fig. 1D), and isolated ALDH1 ${ }^{+}$cells had higher sphere formation capacity than ALDH1 cells (Fig. 1E).

Breast cancer stem-like cell are sensitized to tamoxifen-induced apoptosis and self-renewal inhibition by Let-7c overexpression. The strategy of acquiring mammospheres and the representative images of spheres from different groups are presented in Fig. 2A and B. Tamoxifen decreased the ratio of mammospheres produced from estrogen-treated breast cancer cells (Fig. 2B and C). However, the effect of tamoxifen was reduced when shRNA-ER was introduced (Fig. 2D), indicating that the expression of ER is critical for tamoxifen-induced effects on self-renewal regulation. Let-7c greatly facilitated the tamoxifen functions, however the ER inhibition by shRNA did not exhibit improved anticancer effects of combined application of tamoxifen and Let-7c (Fig. 2D), suggesting the way Let-7c sensitized tamoxifen was dependent on ER.

Tamoxifen induced apoptosis could be strengthened by Let-7c and required ER. Mammospheres isolated from breast cancer cells were successfully infected by Let-7c lentiviral plasmid [lentiviral vector lentilox3.7 (pLL3.7)], and exhibited higher Let-7c expression level than the scramble group (Fig. 3A). Enforced Let-7c induced increased apoptosis (Fig. 3B) and cell cycle arrest compared with the scramble control (Fig. 3C). Tamoxifen stimulated apoptosis of estrogen-treated stem-like breast cancer cells (Fig. 3D). Enforced Let-7 did not increase the apoptosis when ER level was inhibited, and did not sensitize stem-like cells to tamoxifen induction of apoptosis, indicating that Let-7c functions was relying on ER inhibition (Fig. 3E and F), as were illustrated in Fig. 3G.

Let-7c sensitized the breast CSCs through ER-dependent Wnt signaling inhibition. Let-7 family was previously reported to function through post-translational regulation of ER $(8,13)$, and Let-7c decreased ER expression by binding to the 3'untranslated region of its mRNA (13). In the current study, enforced Let-7c expression exhibited suppressive effects on ER and its downstream Wnt signaling activity in spheres from breast cancer (Fig. 4A). Tamoxifen induced the same effects in a similar manner to Let-7c, however with weaker suppressive effects (Fig. 4B). TCF-4 responsive luciferase promoter reporter assay was performed by using pCMV-Green Renilla luciferase assay and Pierce Luciferase Cell Lysis Buffer, and the luciferase activity was normalized to the Renilla luciferase activity. Wnt signaling activity was detected by using a $\beta$-catenin/wild-type TCF-4 responsive luciferase promoter reporter, and results showed the suppressive effects of both Let-7c and tamoxifen exerted on Wnt activity (Fig. 4C). When in combination, stronger inhibition was detected in the group treated with combined Let-7 and tamoxifen, suggesting the assistant roles of Let-7 in tamoxifen induced Wnt blocking (Fig. 4C). To further explore the suppressive Let-7 functioning in estrogen and tamoxifen regulation of stem cells potency, Sox- 2 and Oct- 4 were detected, and the results demonstrated that Let-7c affected the protein expression of the pluripotency stimulators, and the combined usage of Let-7c and tamoxifen reduced the expression of these marker more efficiently than either alone (Fig. 4D). 

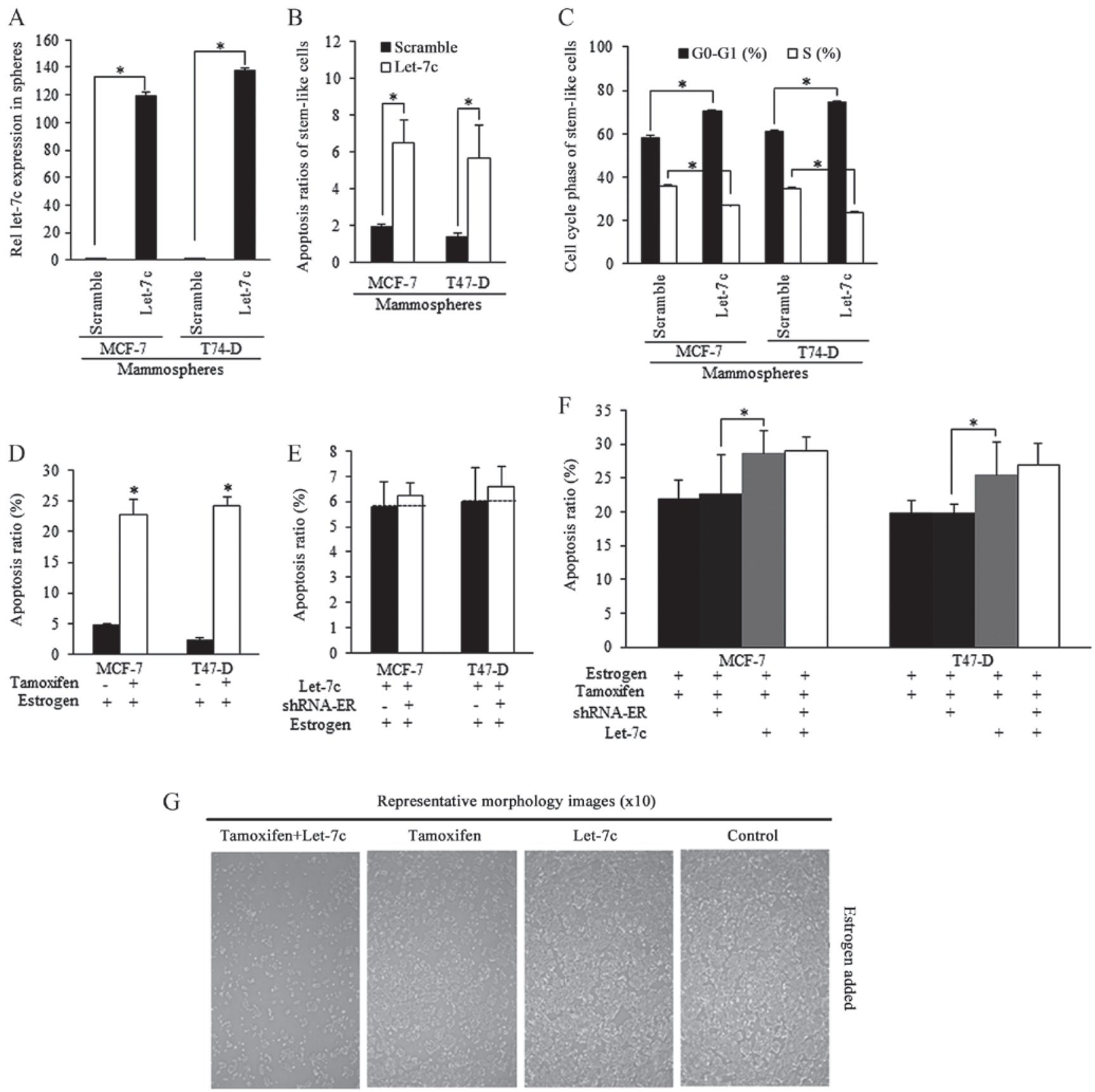

Figure 3. Tamoxifen-induced apoptosis of stem-like cells relies on estrogen inhibition and is be strengthened by Let-7c overexpression dependent on ER Mammospheres isolated from breast cancer cells infected with Let-7c lentiviral plasmid exhibited (A) higher Let-7c expression level than the scramble group, and (B) increased apoptosis and (C) cell cycle arrest. (D) Tamoxifen stimulated apoptosis of stem-like breast cancer cells. (E) Enforced Let-7 did not increase the apoptosis when ER was knocked down using shRNA, indicating that the effects of Let-7c are dependent on ER. (F) Let-7c stimulated the tamoxifen-induced apoptosis of breast cancer stem-like cells, however, combined ER inhibition did not promote the Let-7c stimulated apoptosis further, indicating the way Let-7 acted in induction of apoptosis of tamoxifen treated breast CSCs was dependent on the ER status. (G) Representative images of apoptotic morphology of stem-like cells from different groups (magnification, $\mathrm{x} 100$ ). shRNA, short hairpin RNA; ER. estrogen receptor; $\mathrm{n}=3$ repeats with similar results. Values are presented as the means \pm SEM.

Let-7c inhibits tumor formation and growth of breast CSCs in vivo. To investigate the role of Let- $7 \mathrm{c}$ in inhibiting the growth of ER-positive breast CSCs in vivo, estrogen-treated MCF-7 stem cells that stably overexpressed Let-7c were subcutaneously implanted into immune-deficient mice to form tumors. Tumor size was measured every 5 days. Results demonstrated that overexpressed Let-7c inhibited tumor growth and weight (Fig. 5A; Table I). To identify the effects of Let-7c on MCF-7 stem-like cells in tumor formation, a cell dilution assay was performed in vivo, and Let-7c-induced inhibition of self-renewal was assessed. The end point of observation was tumor emergence within 30 days after implantation at any time-point. The number of tumor occurrence in nude mice was recorded and orresponding to stem cells' renewal, but the tumor growth was not included in the observation. Using the mouse models, Let-7c was demonstrated to decrease tumor formation ability by reducing the self-renewal ability of MCF-7 stem-like cells (Table II). Let-7c inhibits the self-renewal ability following the estrogen treatment, increasing the number of stem cells required for tumor formation in mice with the comparison being 1:398.2 with 1:4,832.6. 
A

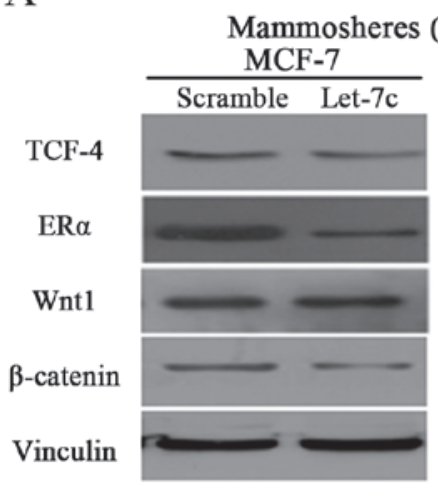

B

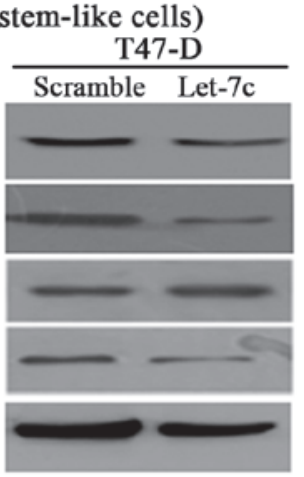

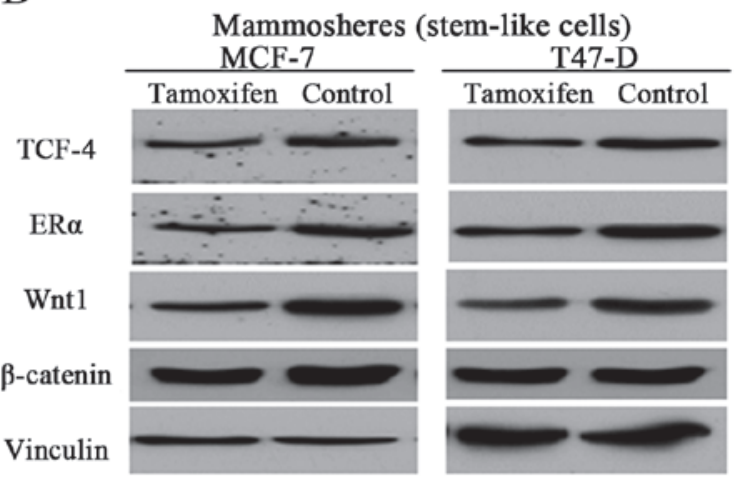

C

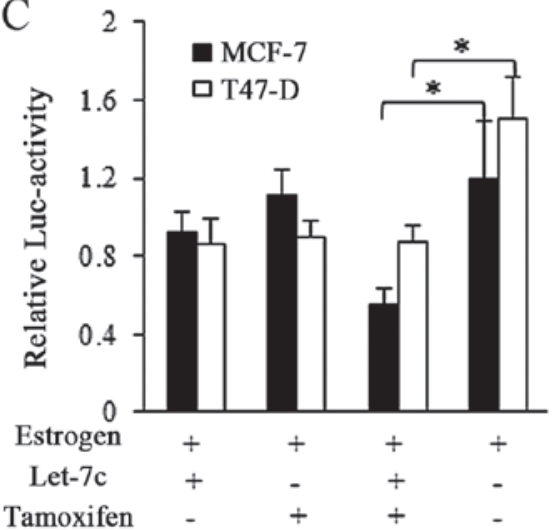

$\mathrm{D}$

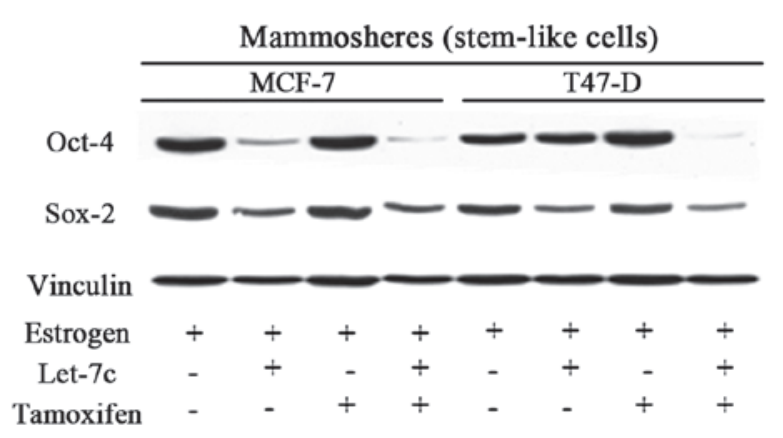

Figure 4. Cooperative functions of combined Let-7c and tamoxifen require ER and suppress Wnt signaling. (A) Let-7c overexpression in mammospheres reduced the expression of ER $\alpha$ and the key factors of Wnt signaling. (B) Tamoxifen functioned the manner as Let-7c with weaker suppressive effect. (C) The wild-type TCF-4 responsive luciferase promoter reporter assay was performed by using pCMV-Green Renilla luciferase assay and Pierce Luciferase Cell Lysis Buffer, and the activity was normalized to the Renilla luciferase activity. (D) Combined Let-7c and tamoxifen inhibited the Wnt signaling activity, however, the individual application of either Let-7c or tamoxifen failed to. TCF-4, T cell factor-4; ER, estrogen receptor; Oct-4, POU domain class 5 transcription factor 1; Sox-2, transcription factor SOX-2 ( $n=3$ repeats with similar results. Values are presented as the means \pm SEM).

ER/Wnt signaling activity is involved in Let-7c-induced functions. To further identify the different expression levels of ER and Wnt signaling pathway downstream components in the murine tumors, mRNA levels by RT-qPCR. The results demonstrated that ER and signaling factors of Wnt were downregulated in the Let-7 group (Fig. 5B). IHC staining confirmed the downregulation of ER, $\beta$-catenin and Wntl proteins (Fig. 5C), suggesting that Let-7 may inhibit the self-renewal of stem-like cells and decrease the tumor formation efficiency of stem cells by suppressing ER and Wnt signaling.

\section{Discussion}

Breast cancer is the leading cause of cancer-associated mortality in women worldwide, due to its natural malignancy and long-term recurrence with acquired drug resistance (26). Improved and complex strategies of therapeutics aiming at eliminating breast cancer presented a promising condition using any or all of the following in patients of different clinical/pathological phages: Surgeries, chemotherapies, radiotherapies, targeted agents, hormonal therapies and biotherapies (8). Tamoxifen is an anti-estrogen drug that binds to ERs and blocks estrogen signaling, acting as a competitive antagonist $(27,28)$. Although it has been applied in clinical treatment, it is only effective in $49 \%$ of patients with ER-positive breast cancer. The association tamoxifen and
miRNAs has been reported in certain previous studies (29-31), in addition to in-depth research into the role of miRNAs and other non-coding RNAs in sensitization to therapeutics (32-34). Cancer stem cells within a tumor prefer to stay at silent mitosis phase, and therefore the lower proliferation index, resulted in resistance to therapies aiming at eliminating the proliferative cells (7). Let-7 exerted inhibitory effects on stem cell renewal, which may control the ratio of stem-like cells in a tumor, which are involved in tumor emergence and recurrence. The mechanisms through which Let-7 functions were reviewed previously (12); however, with the full functions of Let-7 are not established. The current study explored the potential role of Let-7 in sensitization to therapeutic agents, based on previous studies on cancer malignancy and stem cell features. The results will increase our understanding of the potential practical applications of Let-7. Tamoxifen was demonstrated to inhibit cancer proliferation, blocking the estrogen-stimulated stem cell renewal, and Let-7c sensitized CSCs to the effects of tamoxifen via co-regulation of ESR1, in an ESR1 dependent manner. The addition of Let-7c strengthened the suppressive effect of tamoxifen on cancer and renewal activity. In vivo, Let-7 was demonstrated to act as suppressor via Let-7c/ESR1/Wnt signaling, as previously identified in vitro. Additionally, Let-7c suppressed the renewal ability of stem cells, illustrated in the cells dilution assay in vivo, particularly demonstrating the association of stem cell self-renewal with tumor formation. The finding of 
Table II. Frequency of different stem cell suspensions in forming tumors in the mice.

\begin{tabular}{|c|c|c|c|c|c|c|c|c|}
\hline \multirow[b]{2}{*}{ MCF-7 cell suspensions } & \multicolumn{6}{|c|}{ Stem cell number } & \multicolumn{2}{|c|}{ SC frequency } \\
\hline & $10^{6}$ & $10^{5}$ & $10^{4}$ & $10^{3}$ & $10^{2}$ & 50 & Estimate & Limits \\
\hline Scramble-E2 & $7 / 7$ & $10 / 10$ & $7 / 9$ & $4 / 11$ & $0 / 6$ & $0 / 7$ & $1: 398.2$ & $1: 250^{\mathrm{a}}$ \\
\hline Let-7c-E2 & $9 / 9$ & $8 / 9$ & $5 / 13$ & $1 / 10$ & $0 / 9$ & $0 / 7$ & $1: 4,832.6$ & $1: 1,000$ \\
\hline
\end{tabular}

${ }^{a}$ Matching analysis of the different ratios of two groups were performed by two-way repeated measures analysis of variance, and the matching results are statistically significant. F-value, 9.716; P-value, 0.0045.
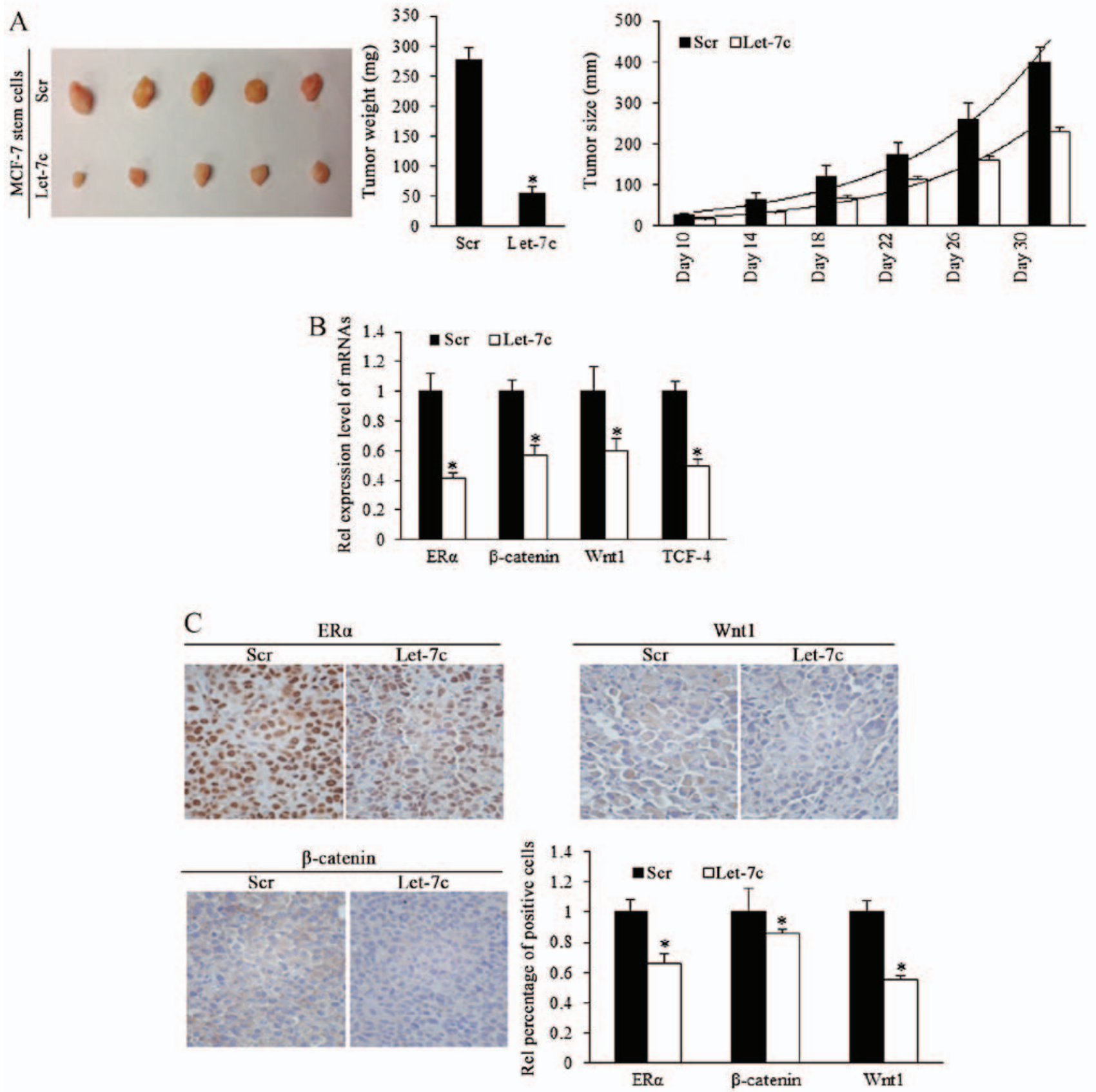

Figure 5. In vivo model of stem-like cells' formation of tumor and the effects of Let-7c on blocking the Wnt activity in tumor growth. (A) Tumor growth curve and tumor weight calculation revealed that Let-7c overexpression of stem-like cells significantly inhibited tumor growth in vivo (Scr, $\mathrm{n}=5$; Let-7c, $\mathrm{n}=5$ ). Scale bar, $1 \mathrm{~cm}$ ). (B) Expression level of ER $\alpha$ and Wnt signaling factors in tumors arising from Let-7c overexpressed group was significantly lower than that arising from miR-control group, as determined by reverse transcription-quantitative polymerase chain reaction. (C) Representative images of Let-7c estrogen receptor and Wnt signaling factors in tumor tissues ( $\mathrm{n}=3$ repeats with similar results; magnification, $\mathrm{x} 400$ ). Statistical significance was evaluated by using Kruskal-Wallis test or analysis of variance. ${ }^{*} \mathrm{P}<0.05$ vs. Scr. Scr, scramble; ER, estrogen receptor; TCF-4, T cell factor. Values are presented as the means \pm SEM. 
the current study and previous research indicate that Let-7 has important functions in breast cancer occurrence, recurrence and stem cell renewal, which may provide a solid research foundation for further clinical applications of ncRNA in breast cancer, and expand the interactions among non-coding RNAs in functional biology, yielding comprehensive understandings of the intracellular genes regulation.

\section{Acknowledgements}

The authors acknowledge assistants in the Key Laboratory (Ministry of Education) of Environment and Genes Related to Disease, Medical College of Xi'an Jiaotong University, the Center for Translational Medicine, the First Affiliated Hospital of Xi'an Jiaotong University (Xi'an, China), and the Institute of Urinary Surgery (the Key Laboratory, Ministry of Education), the First Affiliated Hospital of Xi'an Jiaotong University. Professor Peijun Liu (Center for Translational Medicine, the First Affiliated Hospital of Xi'an Jiaotong University) has exerted his utmost effort in conducting and performing the studies, and his contribution is appreciated. This study was supported by National Science Foundation for Young Scientists of China (grant no. 81602597) referred to Dr Xin Sun, National Natural Science Foundation of China, (grant no. 81272418) referred to Professor Hong Ren. This study was also supported in part by National Science Foundation for Young Scientists of China (grant no. 81402506) referred to Dr Sida Qin.

\section{Competing interests}

The authors declare there is no competing interest.

\section{References}

1. Siegel RL, Miller KD and Jemal A: Cancer statistics, 2015. CA Cancer J Clin 65: 5-29, 2015.

2. Roy V and Perez EA: Biologic therapy of breast cancer: focus on co-inhibition of endocrine and angiogenesis pathways. Breast Cancer Res Treat 116: 31-38, 2009.

3. Geisler J: Differences between the non-steroidal aromatase inhibitors anastrozole and letrozole - of clinical importance? Br J Cancer 104: 1059-1066, 2011.

4. Brodie A, Sabnis G and Macedo L: Xenograft models for aromatase inhibitor studies. J Steroid Biochem Mol Biol 106: $119-124,2007$.

5. Nabholtz JM, Mouret-Reynier MA, Durando X, Van Praagh I, Al-Sukhun S, Ferriere JP and Chollet P: Comparative review of anastrozole, letrozole and exemestane in the management of early breast cancer. Expert Opin Pharmacother 10: 1435-1447, 2009.

6. Miller WR and Larionov AA: Understanding the mechanisms of aromatase inhibitor resistance. Breast Cancer Res 14: 201, 2012.

7. Sun X, Jiao X, Pestell TG, Fan C, Qin S, Mirabelli E, Ren H and Pestell RG: MicroRNAs and cancer stem cells: the sword and the shield. Oncogene 33: 4967-4977, 2014.

8. Sun X, Qin S, Fan C, Xu C, Du N and Ren H: Let-7: a regulator of the ER $\alpha$ signaling pathway in human breast tumors and breast cancer stem cells. Oncol Rep 29: 2079-2087, 2013.

9. Sun X, Tang S-C, Xu C, Wang C, Qin S, Du N, Liu J, Zhang Y, Li X, Luo G, et al: DICER1 regulated let-7 expression levels in p53-induced cancer repression requires cyclin D1. J Cell Mol Med 19: 1357-1365, 2015.

10. Xu C, Sun X, Qin S, Wang H, Zheng Z, Xu S, Luo G, Liu P, Liu J, Du N, et al: Let-7a regulates mammosphere formation capacity through Ras/NF- $\mathrm{kB}$ and Ras/MAPK/ERK pathway in breast cancer stem cells. Cell Cycle 14: 1686-1697, 2015.

11. Sun X, Jiang S, Liu J, Wang H, Zhang Y, Tang SC, Wang J, Du N, Xu C, Wang C, et al: $\mathrm{miR}-208$ a stimulates the cocktail of SOX 2 and $\beta$-catenin to inhibit the let-7 induction of self-renewal repression of breast cancer stem cells and formed miR208a/let-7 feedback loop via LIN28 and DICER1. Oncotarget 6: 32944-32954, 2015.

12. Sun X, Liu J, Xu C, Tang SC and Ren H: The insights of Let-7 miRNAs in oncogenesis and stem cell potency. J Cell Mol Med 20: 1779-1788, 2016
13. Sun X, Xu C, Tang SC, Wang J, Wang H, Wang P, Du N, Qin S, $\mathrm{Li} \mathrm{G}, \mathrm{Xu} \mathrm{S}$, et al: Let-7c blocks estrogen-activated Wnt signaling in induction of self-renewal of breast cancer stem cells. Cancer Gene Ther 23: 83-89, 2016.

14. Wang T, Liu Z, Guo S, Wu L, Li M, Yang J, Chen R, Xu H, Cai S, Chen $\mathrm{H}$, et al: The tumor suppressive role of CAMK2N1 in castration-resistant prostate cancer. Oncotarget 15: 3611-3621, 2014

15. Livak KJ and Schmittgen TD: Analysis of relative gene expression data using real-time quantitative PCR and the 2(-Delta Delta C(T)) Method. Methods 25: 402-408, 2001.

16. Liu H, Liang Y, Li Y, Li Y, Wang J, Wu H, Wang Y, Tang SC, Chen J and Zhou Q: Gene silencing of BAG-1 modulates apoptotic genes and sensitizes lung cancer cell lines to cisplatin-induced apoptosis. Cancer Biol Ther 9: 832-840, 2010.

17. He J, Wang M, Jiang Y, Chen Q, Xu S, Xu Q, Jiang BH and Liu LZ Chronic arsenic exposure and angiogenesis in human bronchial epithelial cells via the ROS/miR-199a-5p/HIF-1 $\alpha / \mathrm{COX}-2$ pathway. Environ Health Perspect 122: 255-261, 2014.

18. Wang T, Guo S, Liu Z, Wu L, Li M, Yang J, Chen R, Liu X, Xu H, Cai S, et al: CAMK2N1 inhibits prostate cancer progression through androgen receptor-dependent signaling. Oncotarget 5: 10293-10306, 2014.

19. Qin S, Yang C, Zhang B, Li X, Sun X, Li G, Zhang J, Xiao G, Gao X, Huang G, et al: XIAP inhibits mature Smac-induced apoptosis by degrading it through ubiquitination in NSCLC. Int J Oncol 49: 1289-1296, 2016.

20. Qin S, Xu C, Li S, Wang X, Sun X, Wang P, Zhang B and Ren $\mathrm{H}$ : Hyperthermia induces apoptosis by targeting survivin in esophageal cancer. Oncol Rep 34: 2656-2664, 2015.

21. Sun Y, Wang Y, Fan C, Gao P, Wang X, Wei G and Wei J: Estrogen promotes stemness and invasiveness of ER-positive breast cancer cells through Gli1 activation. Mol Cancer 13: 137, 2014.

22. Ginestier C, Hur MH, Charafe-Jauffret E, Monville F, Dutcher J, Brown M, Jacquemier J, Viens P, Kleer CG, Liu S, et al: ALDH1 is a marker of normal and malignant human mammary stem cells and a predictor of poor clinical outcome. Cell Stem Cell 1: 555-567, 2007.

23. Liu SY and Zheng PS: High aldehyde dehydrogenase activity identifies cancer stem cells in human cervical cancer. Oncotarget 4: 2462-2475, 2013.

24. Wang T, Liu Z, Guo S, Wu L, Li M, Yang J, Chen R, Xu H, Cai S, Chen $\mathrm{H}$, et al: The tumor suppressive role of CAMK2N1 in castration-resistant prostate cancer. Oncotarget 5: 3611-3621, 2014.

25. Wang T, Guo S, Liu Z, Wu L, Li M, Yang J, Chen R, Liu X, Xu H, Cai S, et al: CAMK2N1 inhibits prostate cancer progression through androgen receptor-dependent signaling. Oncotarget 5: 10293-10306, 2014.

26. Dhami GK, Liu H, Galka M, Voss C, Wei R, Muranko K, Kaneko T, Cregan SP, Li L and Li SS: Dynamic methylation of Numb by Set8 regulates its binding to p53 and apoptosis. Mol Cell 50: 565-576, 2013.

27. Renoir J-M, Marsaud V and Lazennec G: Estrogen receptor signaling as a target for novel breast cancer therapeutics. Biochem Pharmacol 85: 449-465, 2013.

28. Maczis M, Milstien S and Spiegel S: Sphingosine-1-phosphate and estrogen signaling in breast cancer. Adv Biol Regul 60: $160-165,2016$

29. Ward A, Shukla K, Balwierz A, Soons Z, König R, Sahin O and Wiemann S: MicroRNA-519a is a novel oncomir conferring tamoxifen resistance by targeting a network of tumour-suppressor genes in $\mathrm{ER}^{+}$breast cancer. J Pathol 233: 368-379, 2014.

30. Ward A, Balwierz A, Zhang JD, Küblbeck M, Pawitan Y, Hielscher T, Wiemann S and Sahin Ö: Re-expression of microRNA-375 reverses both tamoxifen resistance and accompanying EMT-like properties in breast cancer. Oncogene 32: $1173-1182,2013$.

31. Miller TE, Ghoshal K, Ramaswamy B, Roy S, Datta J, Shapiro CL, Jacob S and Majumder S: MicroRNA-221/222 confers tamoxifen resistance in breast cancer by targeting $\mathrm{p} 27^{\mathrm{Kipl}}$. J Biol Chem 283: 29897-29903, 2008

32. Han Li C and Chen Y: Small and long non-coding RNAs: novel targets in perspective cancer therapy. Curr Genomics 16: 319-326, 2015.

33. Crea F, Watahiki A, Quagliata L, Xue H, Pikor L, Parolia A. Wang Y, Lin D, Lam WL, Farrar WL, et al: Identification of a long non-coding RNA as a novel biomarker and potential therapeutic target for metastatic prostate cancer. Oncotarget 5: 764-774, 2014.

34. Chen ZZ, Huang L, Wu YH, Zhai WJ, Zhu PP and Gao YF: LncSox4 promotes the self-renewal of liver tumour-initiating cells through Stat3-mediated Sox4 expression. Nat Commun 7: $12598,2016$.

This work is licensed under a Creative Commons Attribution-NonCommercial-NoDerivatives 4.0 International (CC BY-NC-ND 4.0) License. 